\title{
Analysis On The Spatial Differentiation Characteristics of Poverty Risk Caused By Disaster Under The Stress of Geological Disasters - A Case Study of Sichuan Province
}

Mingshun Xiang ( $\nabla$ xiangmingshun19@cdut.edu.cn )

Chengdu University of Technology

Linsen Duan

Chengdu University of Technology

Fengran Wei

Chengdu University of Technology

Jin Yang

Chengdu University of Technology

Wenheng Li

Chengdu University of Technology

Chunjian Wang

Chengdu University of Technology

Wenbo Yang

Chengdu University of Technology

Qiuchi Deng

South China Normal University

\section{Research Article}

Keywords: Geological Disasters, Poverty Caused by Disaster, Sustainable Development, Spatial Pattern, Sichuan Province

Posted Date: December 22nd, 2021

DOI: https://doi.org/10.21203/rs.3.rs-1046221/v1

License: (1) This work is licensed under a Creative Commons Attribution 4.0 International License.

Read Full License 
Version of Record: A version of this preprint was published at Environmental Science and Pollution Research on March 7th, 2022. See the published version at https://doi.org/10.1007/s11356-022-19485-4. 
1 Analysis on the Spatial Differentiation Characteristics of Poverty

2 Risk Caused by Disaster under the Stress of Geological Disasters

3 - A Case Study of Sichuan Province

4

Mingshun Xiang ${ }^{1,3}$, Linsen Duan², Fengran $\mathrm{Wei}^{1}$, Jin Yang ${ }^{1,2,3^{*}}$, Wenheng $\mathrm{Li}^{1}$, Chunjian Wang, Wenbo Yang ${ }^{3}$ and Qiuchi Deng ${ }^{4}$

${ }^{1}$ College of Tourism and Urban-Rural Planning, Chengdu University of Technology, Chengdu 610059, China

${ }^{2}$ College of Earth Science, Chengdu University of Technology, Chengdu 610059, China

${ }^{3}$ Research Center for Human Geography of Tibetan Plateau and its Eastern Slope (Chengdu University of Technology), Chengdu 610059, China

${ }^{4}$ School of Geography, South China Normal University, Guangzhou 510631, China

E-mail: yangj8872@163.com

Keywords: Geological Disasters, Poverty Caused by Disaster, Sustainable Development, Spatial Pattern; Sichuan Province

\section{Abstract}

Research on the poverty risk caused by disasters in disaster - prone areas is a useful exploration to coordinate social economic development with disaster prevention and reduction, and is of great significance to regional sustainable development. Based on statistical data and spatial data, this paper takes Sichuan Province as the typical research area. Remote sensing and geographic information technology are used to study the poverty risk caused by disasters based on the quantitative evaluation of geological disasters risk and regional development level. the spatial differentiation characteristics of poverty risk caused by disasters are explored on the $1 \mathrm{~km} \times 1 \mathrm{~km}$ grid scale. The results indicate that: (1) The overall risk of geological disasters in Sichuan Province is relatively high, with high and relatively high risk areas accounting for more than $40 \%$, low and relatively low risk areas accounting for less than $30 \%$. The risks in Mountain and Ravine Areas are significantly higher than other areas. (2) The regional development level in Sichuan Province is relatively high, but with significant the spatial differences. The development level of high-altitude areas and remote mountainous areas is quite different from that of the Chengdu Plain in the middle Sichuan Province. the problem of uneven development in the east, middle, and west is prominent. (3) The poverty risk caused by disasters is high, and the spatial pattern presents a characteristic of "high in the west and low in the east" with high positive spatial correlation. High - High Cluster Areas are mainly distributed in western and southwestern Sichuan. Low - Low Outlier Areas are mainly distributed in Chengdu Plain and Hilly Areas of Sichuan Basin. High - Low Outlier and Low - High Outlier Areas occupy a relatively small percentage with scattered distribution. This paper provides a reference for the coordinated management of disaster prevention and reduction, as well as social and economic development in underdeveloped areas.

\section{Introduction}


Looking back on the history of world development, natural disasters, diseases and epidemics have always been accompanied by the development of human society, resulting in a large number of casualties and heavy economic losses ( $\mathrm{Li}$ et al 2020). China is a mountainous country with mountain areas accounting for $69.4 \%$ of the total land, and population in those areas accounting for more than one-third of the whole population. With complex and diverse geographical environments, the unique energy gradient in the mountain area usually induced geological disasters such as debris flow, landslide and collapse, causing serious loss of people's lives and property accompany by restricting regional development (Cui 2014). Due to geographical constraints, the economic development of mountain areas is lagging. All the 14 contiguous poverty-stricken areas in China before 2020 are in mountain areas, where low-income people are concentrated. The fragile ecological environment, frequent disasters and backward social as well as economic development have led to $20 \%$ of China's poor farmers being impoverished caused by disasters, which is ranking second among the factors causing poverty. Through the fight against poverty, China has eliminated absolute poverty and achieved remarkable achievements in disaster prevention, mitigation and relief to reduce poverty. And now the governance of relative poverty will focus on the work of agriculture, rural areas and farmers.

Recently, more and more scholars begin to pay attention to the relationship between poverty and disaster. Human and economic vulnerability to related disasters is highly related to economic development level (Wu et al 2018). World economic and social development has greatly promoted the human poverty reduction. However, disasters, wars, climate change and other factors have seriously restricted the process of global sustainable poverty reduction ( $\mathrm{Li}$ et al 2020). From the perspective of the relationships among economic poverty, disaster risk and environmental degradation, economic poverty is the external driving force of the vicious cycle of "frequent disaster-ecological degradation-poverty intensification" (Ding et al 2013, Andrew and Mikhail 2017). Disasters will have direct or indirect short-term or long-term impacts and injuries on different levels of countries, regions, families, individuals, etc., especially on rural families in developing countries (Gignoux and Menéndez 2016). The asset stock of low-income families in disaster-prone areas is not enough and being vulnerable, which not only makes their assets vulnerable to lose, but also seriously worsens their income sources in the future, and then affects their post-disaster reconstruction and recovery capacity thus makes them fall into the persistent poverty trap (Bidisha et al 2021, Sakai et al 2017, Carter and Barrett 2006).

In recent years, scholars have made positive progress on the coupling among geographic patterns of poverty, poverty and geographic environmental factors (Berberich 2019; Elwood et al 2017; Jessie et al 2016, Zhou 2021, Li et al 2020). Poverty has a geographical spatial attribute (Besagni and Borgarello 2019, Iparraguirre 2012). Bird and Shepherd (2003) Pointed that the spatial poverty trap is the area with a small stock of "geographic capital" and high incidence of poverty. There is a coupling between economic poverty, disaster risk and environmental degradation.the poor tend to be concentrated in remote areas with poor natural environment,backward infrastructure and public service capacity (Ding et al 2013, Zhou 2021). The economic structure of agriculture in poverty- 
stricken areas is relatively homogeneous, and the livelihood of farmer is extremely dependent on natural resources and ecosystem services. so, the geographical distribution of environmental vulnerability and poverty is highly coupled (Barbier 2010). Disasterprone areas have stronger social vulnerability, it is crucial to rely on community strength to cope with disasters (Kirby et al 2019, Rampengan et al 2014). Given this, some scholars have studied the relationship between the threat of geological disasters and the willingness of farmers relocation and discuss the livelihood issues of farmers under the stress of geological disasters (Xu et al 2017).

At present, most scholars focus on the natural attributes of geological disasters, but lacking researches on the social attributes between disasters and regional development, especially the quantitative evaluation from the geospatial perspective. Therefore, this paper is taking Sichuan Province as an research interest, and construct a geological disaster poverty risk evaluation model based on the relationship between disasters and regional development. the paper also quantitatively studies the poverty risk caused by disasters and its geospatial pattern in Sichuan Province, and provide a theoretical basis for coordinated management and administration of geological disasters and relative poverty.

\section{Study Area and Data Sources}

\subsection{Overview of the Study Area}

The geographical environment of Sichuan Province is complex, mainly mountains, plateaus and hills. The geological structure is changeable, the neotectonics is active, the geological disasters are frequent, and the spatial distribution types and development characteristics are different. The total area of geological disaster-prone areas accounts for $97 \%$ of the total land area of Sichuan province, which is one of the provinces with the most severe geological disasters in China. At the same time, Sichuan has four contiguous poverty-stricken areas before 2020, namely, the Qinba Mountains, the Wumeng Mountains, the Yi Area in Liangshan Mountain and the Tibetan-related areas in Sichuan, along with 88 poverty-stricken counties. At the end of 2013 , the rural poverty population was 6.25 million, making it one of the impoverished counties and provinces with the most impoverished population in China.

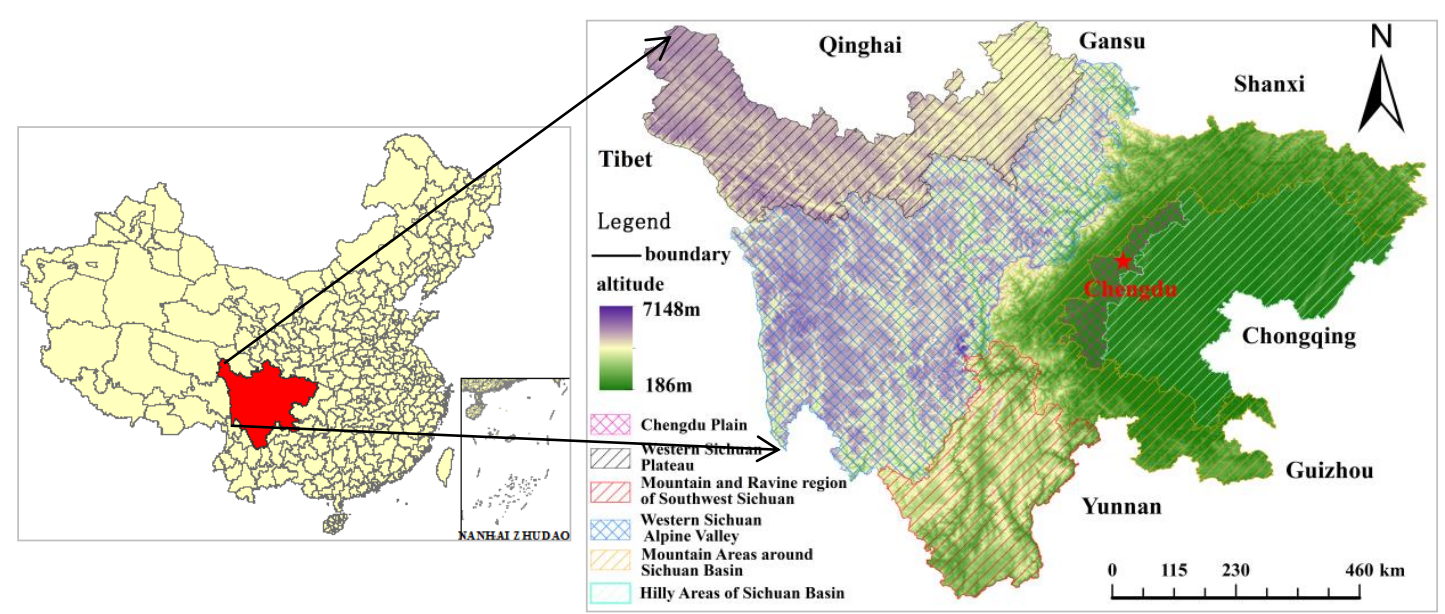

Figure 1 The Location of Sichuan Province

\subsection{Data Sources and Processing}


The data used in this paper mainly include spatial data, socioeconomic data and demographic data as follows. (1) Spatial data: 90m resolution DEM and $250 \mathrm{~m}$ vegetation index data from Geospatial Data Cloud (http://www.gscloud.cn/); data of 35047geological disaster points are from Resources and Environmental Science and Data Center (http://www.resdc.cn/); engineering geological rock groups that classified according to the 1:500000 digital geological map of Sichuan Province; rainfall and other meteorological data from China Meteorological Data Network, (http://data. cma.cn); data on arable land, residential area, river system from Sichuan Land Use Change Data in 2018; traffic vector data from Geographic Data Sharing Infrastructure, College of Urban and Environmental Science, Peking University (https://geodata.pku.edu.cn/). (2) Socioeconomic and demographic data: per capita GDP, per capita disposable income of residents, average salary, number of primary and secondary schools are from the 2019 Sichuan Provincial Statistical Yearbook and the statistical yearbooks of cities; water resources data are from the 2019 Sichuan Provincial Water Resources Bulletin; data on medical and health institutions from 2019 Sichuan Provincial Medical and Health Yearbook.

All the data were entered into GIS geodatabase after preprocessing. Furthermore, the projection and coordinate system of all the data were transformed into UTM84N and WGS84 projection,by GIS software.

\section{Methods}

\subsection{Geological Disaster Risk Assessment}

\subsubsection{Deterministic Coefficient Method}

The amount of information can objectively reflect the contribution of evaluation factors to the risk of geological disasters under different classification standards. The greater the index is, the higher the risk of geological disasters will be. Combined with the content of information, this paper uses the certainty factor (CF) to determine the weight. CF was proposed by Shortliffe and Buchanan (1975), which is usually used for sensitivity analysis of different factors, and gradually used for calculating the weight of each factor (Liang et al 2019). The method is as following:

$$
C F= \begin{cases}\frac{P P_{a}-P P_{s}}{P P_{a}\left(1-P P_{s}\right)} & \left(P P_{a} \geq P P_{s}\right) \\ \frac{P P_{a}-P P_{s}}{P P_{s}\left(1-P P_{a}\right)} & \left(P P_{a} \leq P P_{s}\right)\end{cases}
$$

In this formula, $P P_{a}$ is the ratio of the number of geological disasters in factor $a$ to the area of factor $a$; $P P_{s}$ is the ratio of the number of geological disasters in the study area to the area of the study area. The variation range of $C F$ is $[-1,1]$; a positive value indicates a high certainty of geological disaster occurrence, which is more likely to occur; a negative value represents a lower certainty of geological disaster occurrence, which is less likely to occur; when the calculation result is close to 0 , it means that the factor cannot determine whether the geological disaster is likely to occur in this classification.

The weight $\omega_{i}$ is calculated as follows,

$$
\omega_{i}=C F_{(i, \max )}-C F_{(i, \min )}
$$

In the formula, $C F_{(i, \max )}$ is the maximum value to determine the coefficient in each 
classification of factor $i$, and $C F(i, m i n)$ is the minimum value to determine the coefficient in each classification of factor $i$.

\subsubsection{Risk Assessment Method}

The disaster index is an indicator reflecting the scale of geological disasters in each evaluation unit, and the calculation formula is as follows,

$$
V_{i}=\sum_{i=1}^{n} \omega_{j} \times y_{j} \quad(j=1,2, \Lambda n)
$$

In this formula, $V_{i}$ is the evaluation unit. $V_{i}$ is the comprehensive risk index of geological disasters of the evaluation unit. $\omega_{j}$ is the weight value of the evaluation index $j$ of the evaluation unit $i . y_{j}$ is the normaliezed value of the evaluation index $j$ of the evaluation unit $i$.

\subsubsection{Geological Disaster Risk Assessment Index}

Combining relevant research (Liang et al 2019, Luo et al 2020) and field investigation, the paper comprehensively considers topography, land cover, lithology, meteorology, hydrology. We selects 7 indicators as the risk assessment factors of geological disasters. The evaluation index system is shown in Table 1.

Table 1 Geological Disaster Risk Assessment Index System

\begin{tabular}{cc}
\hline Indicator & Weight $\left(W_{i}\right)$ \\
\hline Slope $\left({ }^{\circ}\right)$ & 0.1673 \\
Relief degree of land surface $(\mathrm{m})$ & 0.1233 \\
Elevation $(\mathrm{m})$ & 0.2205 \\
Vegetation coverage & 0.1077 \\
Engineering geological rock group & 0.1424 \\
Distance from fault $(\mathrm{km})$ & 0.0981 \\
Daily mean maximum precipitation for years $(\mathrm{mm})$ & 0.1407 \\
\hline
\end{tabular}

\subsection{Regional Development Level Evaluation Method}

\subsubsection{Comprehensive Development Index Method}

(1) Comprehensive Development Index

The Comprehensive Development Index (CDI) is used to reflect the comprehensive development level in the study area. the larger the index value, the richer the region will beand vice versa.. The CDI could be calculated as following (Liu et al 2020).

$$
C D I=\sum_{i=1}^{n}\left(\sum_{j=1}^{m} F_{i j} \omega_{i j}\right) \omega_{i}
$$

In the formula: $C D I$ is the comprehensive development index; $F_{i j}$ is the indicator value after standardized processing, $\omega_{i j}$ represents the index weight; $\omega_{i}$ represents the dimension weight; $n$ is the number of dimensions, and $m$ is the number of indicators corresponding to a certain dimension.

(2) Data normalized

The evaluation indicators of CDI include both positive and negative values. 
Therefore, the standardization of data in this paper adopted the extreme difference standard method, and the specific formulas are as follows:

$$
\begin{gathered}
\text { positive indicator: } Y_{\mathrm{ij}}=\frac{X_{i j}-X_{\min }}{X_{\max }-X_{\min }} \\
\text { negative indicator: } Y_{\mathrm{ij}}=\frac{X_{\max }-X_{i j}}{X_{\max }-X_{\min }}
\end{gathered}
$$

In those formulas, $Y_{i j}$ is the indicator value after standardized processing; $X_{i j}$ is the original data of the evaluation index $j$ of the county $i$ in Sichuan Province. $X_{\max }$ and $X_{\min }$ are the maximum and minimum values of the evaluation index $j$, respectively.

\section{(3) Calculation of Index Weight}

The calculation method of index weight can be divided into subjective method and objective method. In order to reduce subjective biases in expert judgments, incomplete data and objective biases caused by data quality problems, the Analytic Hierarchy Process (AHP) and Entropy Method are used to calculate weights, respectively (Ni et al 2009, Chen et al 2009). then the subjective and objective weights are added to get the comprehensive weight.

\subsubsection{Evaluation System}

According to specific research needs, the relationships between research results and socioeconomic development level in Sichuan Province are considered (Alkire and Foster 2011, Schleicher et al, 2018, Jin et al 2020, Liu et al 2020). and we measured CDI from four dimensions (resource, economic, income, education \& medical care) and 18 indicators (Table 2).

\begin{tabular}{|c|c|c|}
\hline Dimension & Indicator & $\begin{array}{c}\text { Combination } \\
\text { weight }\left(W_{i j}\right)\end{array}$ \\
\hline \multirow{4}{*}{ Resource endowment } & $\begin{array}{l}\text { Per capita area of cultivated land area } \\
\qquad\left(\mathrm{hm}^{2} / \text { person }\right)\end{array}$ & 0.0795 \\
\hline & Per capita water resources $\left(\mathrm{m}^{3} /\right.$ person $)$ & 0.0346 \\
\hline & Per capita village area $\left(\mathrm{m}^{2} /\right.$ person $)$ & 0.0497 \\
\hline & Transportation resources $\left(\mathrm{Km} / \mathrm{km}^{2}\right)$ & 0.0720 \\
\hline \multirow{5}{*}{$\begin{array}{c}\text { The level of economic } \\
\text { development }\end{array}$} & Per capita GDP $\left(10^{4}\right.$ yuan $\left.^{¥}\right)$ & 0.0276 \\
\hline & Urbanization rate $(\%)$ & 0.0330 \\
\hline & $\begin{array}{l}\text { General public budget revenue }\left(10^{8}\right. \\
\left.\qquad \operatorname{yuan}^{¥}\right)\end{array}$ & 0.0267 \\
\hline & $\begin{array}{l}\text { Total investment in fixed assets }\left(10^{8}\right. \\
\left.\qquad \text { yuan }^{¥}\right)\end{array}$ & 0.0309 \\
\hline & Employment situation $\left(10^{4}\right.$ people $)$ & 0.0560 \\
\hline \multirow{4}{*}{ Income level } & Per capita disposable income (yuan⿱䒑⿻二丨䒑口) & 0.0644 \\
\hline & average salary (yuan) & 0.0350 \\
\hline & per capita grain output (kg / person) & 0.0797 \\
\hline & Rural poverty incidence (\%) & 0.1966 \\
\hline
\end{tabular}

\section{Table2 CDI Evaluation System}


Number of primary and secondary schools

Teacher-student ratio

0.0216

Education \& medical care

Medical and health institutions

0.0453

service

Number of beds per thousand

registered population (Bed / 1000

0.0478

people)

Health technical personnel (people)

0.0367

\subsection{Evaluation Method of the Poverty Risk Caused by Geological Disaster}

According to the general risk assessment formula, the poverty risk caused by geological disasters consists of geological disaster risk and the regional development level (Tian and Zhang 2016). The formula is as follows:

$$
R_{m}=\sum V_{i} \times C D I_{j}
$$

In this formula, $R_{m}$ is the risk index of poverty caused by geological disasters. The higher the index, the higher the poverty risk caused by disasters is. $V_{i}$ is the risk of geological disasters. $C D I_{J}$ is a regional comprehensive development index.

The evaluation process of poverty risk caused by geological disasters is shown in Figure 2.

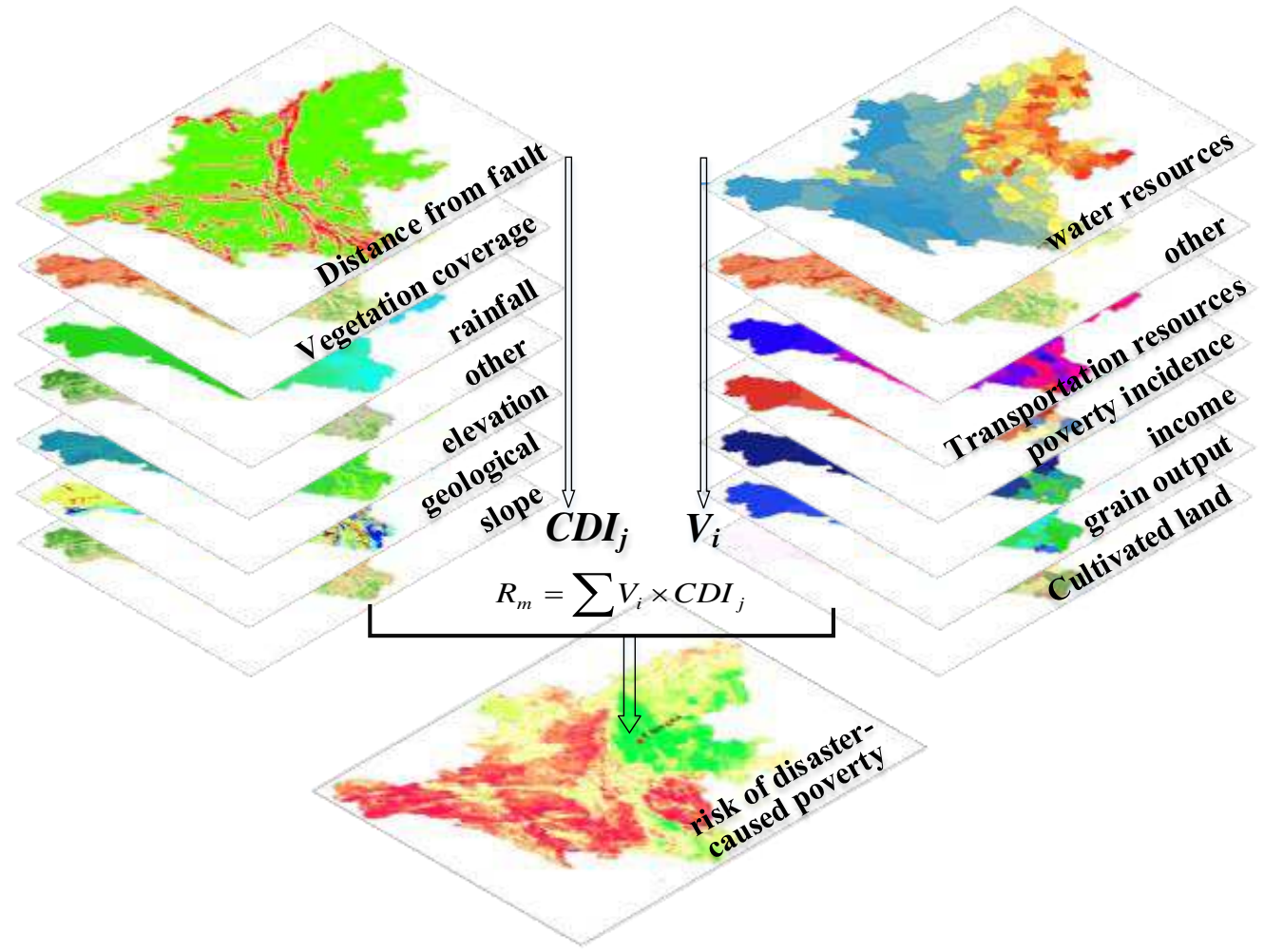




\subsection{Exploratory Spatial Analysis Method}

\subsubsection{Global Spatial Autocorrelation}

Global Moran's I is used to describe the spatial differentiation characteristics of poverty risk caused by disasters in the study area. The formula is as follows:

$$
I=\frac{n \sum_{i=1}^{n} \sum_{j=1}^{n} w_{i, j}\left(x_{i}-\bar{x}\right)\left(x_{j}-\bar{x}\right)}{\sum_{i=1}^{n} \sum_{j=1}^{n} \omega_{i j} \sum_{i=1}^{n}(x i-\bar{x})^{2}}
$$

In this formula, $w_{i j}$ is the spatial weight; $x$ is the mean value of the attribute; $x_{i}$ and $x_{j}$ are the attribute values of the elements $i$ and $j$, respectively; $\mathrm{n}$ is the number of units, and the correlation is considered significant when $|Z|>1.96$.

\subsubsection{Local Spatial Autocorrelation}

Local autocorrelation reveals the local clustering characteristics of spatial unit attributes by analyzing the difference degree and significance level between spatial unit and surrounding units. the formula is as follows:

$$
I_{i}(d)=\frac{n\left(x_{i}-\bar{x}\right) \sum_{j=1}^{n} w_{i j}\left(x_{j}-\bar{x}\right)}{\sum_{i=1}^{n}\left(x_{j}-\bar{x}\right)^{2}}
$$

\section{Results and Analysis}

\subsection{Analysis of Geological Disaster Risk}

Formula1-3, was used to calculate the geological disaster index by GIS software. the study area was classified into five grades by natural breakpoint method: Relatively low risk, Low risk, Moderate risk, High risk and Relatively high risk (Figure 3).

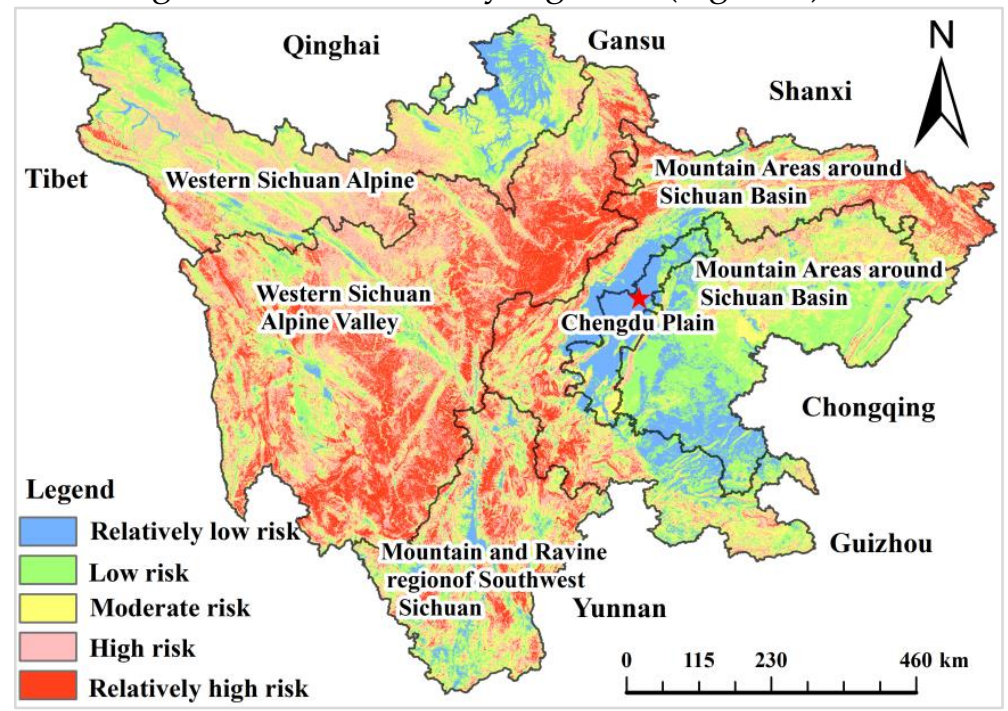

Figure 3 Classification Results of Geological Disaster Risk

As shown in Figure 3, with respect to spatial pattern of geological disaster, the relatively high risk areas are mainly distributed in Western Sichuan Alpine Valley, 
Mountain and Ravine region of Southwest Sichuan and Mountain Areas around Sichuan Basin. High risk areas are less distributed in Chengdu Plain and Hilly Areas of Sichuan Basin, while the other 4 areas have a higher distribution proportion. Similar to high risk areas, the moderate risk areas are less distributed in Chengdu Plain, and the other 5 areas account for a relatively high proportion. The relatively low risk areas are concentrated in Hilly Areas of Sichuan Basin, while the risks in other 5 areas are relatively not significant . The low risk areas are mainly distributed in Chengdu plain and Hilly Areas of Sichuan Basin, while the other 4 areas are sporadically distributed.

the statistics results of the numbers of geological disasters risk areas of various grades are as follows. the area of relatively high risk area is $73574.81 \mathrm{~km}^{2}$, accounts for $14.14 \%$; the area of high risk area is $137037.55 \mathrm{~km}^{2}$, accounts for $28.20 \%$; the area of moderate risk area is $130,263.67 \mathrm{~km}^{2}$, accounts for $26.80 \%$; the area of relatively low risk area is $99829.32 \mathrm{~km}^{2}$, accounts for $20.54 \%$; the area of low risk area is $45294.65 \mathrm{~km}^{2}$, accounts for $9.32 \%$. the risk of geological disasters in Sichuan Province is relatively high in the whole area and widely distributed. its distribution is closely related to the topography and landforms, thus presenting significant geographic spatial differences.

\subsection{Analysis of Regional Development}

considering the county-level administrative divisions as the evaluation unit, the comprehensive development index (CDI) of each region was calculated respectively by GIS software according to formulas 4-6. the development level index of 183 districts and counties was divided into 5 grades through natural breakpoint method (Figure 4), which are High, Relatively high, Medium, Relatively low and Low.

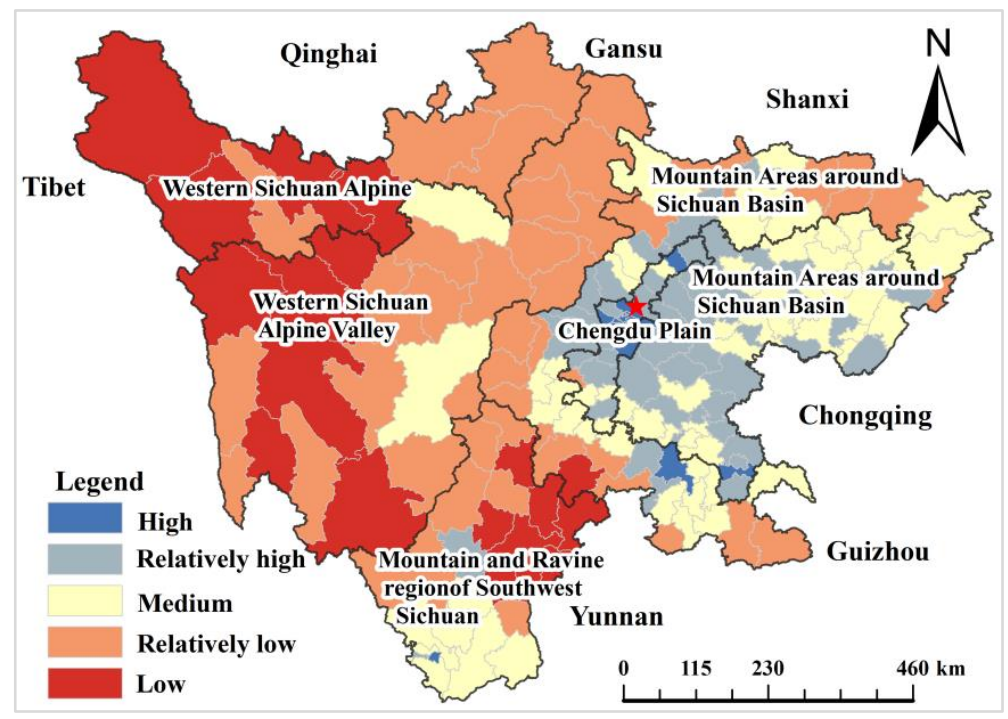

Figure 4 Multidimensional Development Level Grading Results

As shown in Figure 4, the development of every district and county in Sichuan province varies greatly. From the perspective of spatial pattern, the areas with low and relatively low comprehensive development level are mainly located in the Western Sichuan Plateau Area, Mountain and Ravine Area of Western Sichuan and Mountain and Ravine region of Southwest Sichuan. The areas with medium comprehensive development level are mainly located in Hilly Areas of Sichuan Basin and Mountain Areas around 
Sichuan Basin, while the other areas are scattered. The areas with relatively high development level are mainly located in Hilly Areas of Sichuan Basin and Chengdu Plain area; areas with high level development level is relatively small, mostly located in Chengdu Plain Area., the normal distribution characteristics are significant considering the proportions of various development grades. thehigh development level counties accounts for $6.56 \%$ in number; the relatively high development level counties accounts for $25.14 \%$ in number. The number with medium level counties accounts for $33.33 \%$, the number with relatively low level counties accounts for $24.04 \%$, the number with low level counties accounts for $10.93 \%$. In general, with the Chengdu Plain being in the center of Sichan Province, the level of comprehensive development in Sichuan Province is lower as it goes outwards of Chengdu Plain . the level of comprehensive development in mountainous areas and high-altitude areas is obviously low, which is basically consistent with the spatial distribution of 88 counties out of poverty, indicating that these areas will remain the work focus of agriculture, ruralareas and peasantry in the future.

\subsection{Analysis of Poverty Risk Caused by Disasters}

\subsubsection{Overall Characteristics of Poverty Risk Caused by Disasters}

Based on the evaluation of geological disaster risk and regional development, the poverty risk caused by disasters in the study area was calculated. and the natural breakpoint method was used to divide the risk of poverty into 5 grades: High, Relatively high, Medium, Relatively low, and Low (Figure 5). The overall spatial pattern of poverty risk in the study area are high in the west and low in the east. Due to the lag of comprehensive development, higher social and economic vulnerability, and weak ability to resist geological disasters, families' livelihood in Western Sichuan Alpine Valley as well as Western Sichuan Alpine is more vulnerable to geological disasters than that in the central and eastern regions of Sichuan Province. the poverty risk caused by disasters is higher. Instead, cities such as DongDu and Mianyang in the central part, Suining and Nanchong in the east, Yibin and Luzhou in the southeast, and Panzhihua in the south all have relatively low risk of poverty caused by disasters, mainly attribute to their high social and economic level or low risk of regional geological disasters.

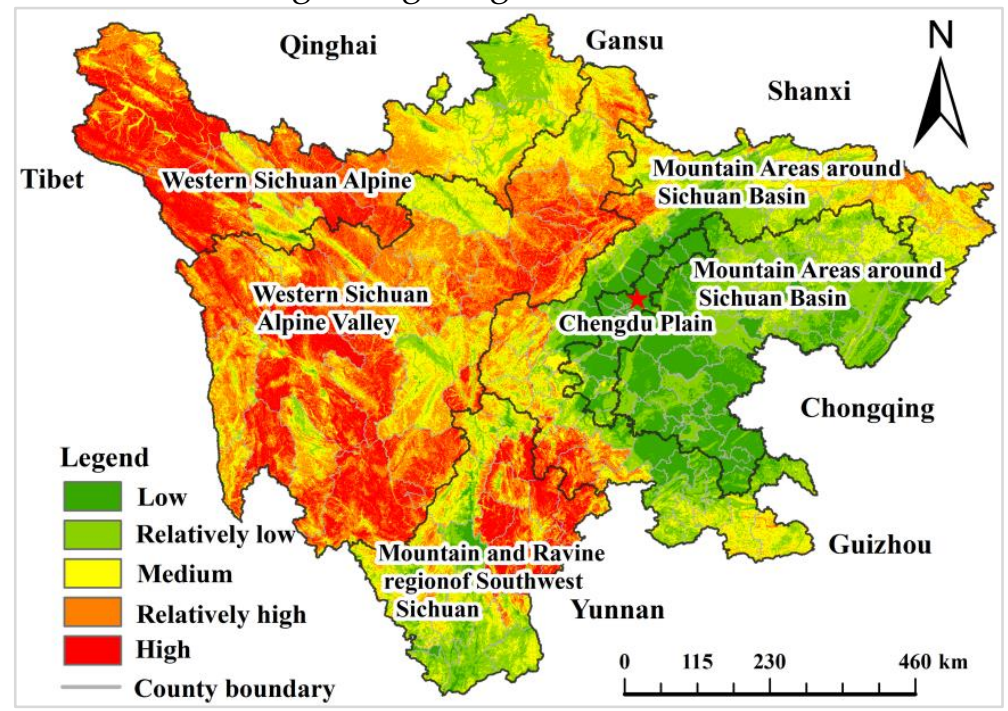


The statistical results of the areas of poverty risk grades caused by disaster are presented in Figure 6. the area of high risk area was $73574.81 \mathrm{~km}^{2}$, and the area of relatively high risk area was the largest, reaching $137037.55 \mathrm{~km}^{2}$. the area of medium risk area is close to that of high risk area, accounting for $130,263.67 \mathrm{~km}^{2}$. the area of low risk area is $99,829.32$ $\mathrm{km}^{2}$. the area of relatively low risk area is account for the smallest at $45,294.65 \mathrm{~km}^{2}$. the results indicate a high overall poverty risk caused by disasters in Sichuan Province.

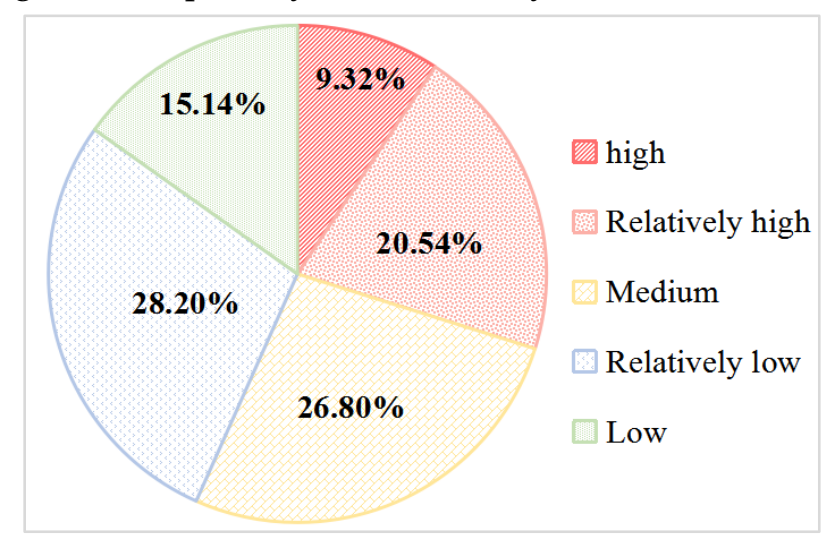

Figure 6 Proportion of Different Grades of Poverty Risk Caused by Disasters in the Study Area

\subsubsection{Spatial Distribution Characteristics of Poverty Risk Caused by Disasters}

The evaluation results of poverty caused by disasters in the study area were gridded with a grid size of $1 \mathrm{~km} \times 1 \mathrm{~km}$, and the Moran's I index at the grid scale is calculated. the Moran's I index of poverty caused by disasters in Sichuan Province is 0.767 , the value of $p<0.001$, indicating that there is a significant positive spatial correlation between disaster and poverty risk in Sichuan Province at the grid scale of $1 \mathrm{~km} \times 1 \mathrm{~km}$. the disaster risk caused by poverty is spatially dependent and clustered. That is, the risk of poverty caused by disasters has regional characteristics.

The local indications of Spatial Association (LISA) is used to study the local clustering characteristics of spatial unit attributes of poverty risk caused by disasters in Sichuan Province, and the spatial clustering is divided into four types. High-High Cluster, research grid and neighboring grids are at high risk of poverty caused by disasters; High-Low Outlier, The risk of poverty caused by disasters in the research grid is high, while the risk of poverty caused by disasters in the neighboring grid is low; Low-High Outlier, The risk of poverty caused by disasters in the research grid is low, and the risk of poverty caused by disasters in the surrounding neighborhood grid is relatively high; Low-Low Cluster, the risk of poverty caused by disaster is low in research grid and neighboring grid. The local spatial autocorrelation of poverty caused by disaster is shown in Figure 7. 


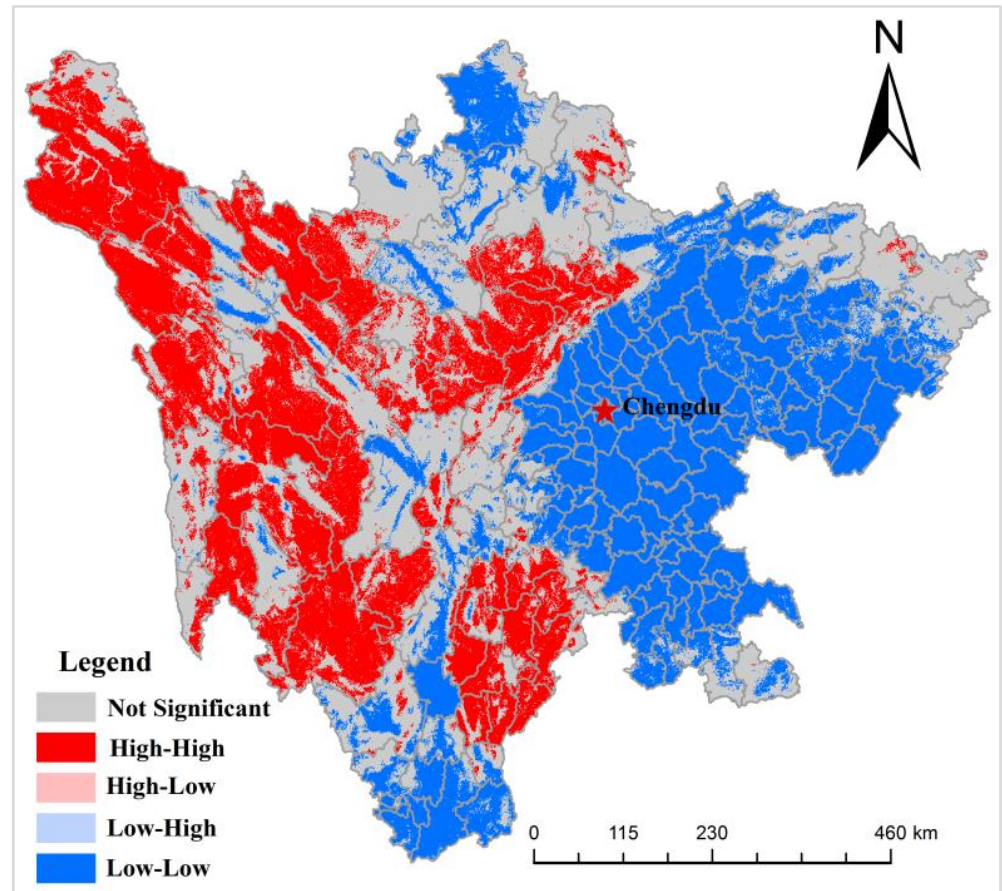

\section{Figure 7 LISA Clustering Map of the Poverty Risk Caused by Disasters in the Study Area}

The results show that the spatial clustering characteristics of poverty risk caused by disasters in Sichuan Province are different, showing a clustering characteristic of "high in the west and low in the east". High-High and Low-Low Cluster is obvious, while the HighLow and Low-High Outlier is not significant. The details are as following.

(1) High-High Cluster Area: With a total area of $142,418.80 \mathrm{~km}^{2}$. the type is mainly distributed in most districts and counties of Ganzi Prefecture, Wenchuan County, Li County and Mao County in Aba Prefecture, and Liangshan Prefecture in western Sichuan. these areas accounts for $29.30 \%$ of the province's land area. Located in the southeastern edge of the Qinghai-Tibetan Plateau, the above three areas, with the main topography of high Mountains, Ravines and Plateaus, are all contiguous poverty-stricken areas in China until 2020. At the same time, geological disasters occur frequently in this area, and the damage intensity is high. In recent years, major natural disasters impact frequently in these areas, such as Wenchuan earthquake, Xinmo Village high-position landslidein Diexi Town, Wenchuan torrent and debris flow, Danba County torrent and debris flow. The interweaving of development lagging and the geological disasters is the most important characteristics in High-High Cluster Area.

(2) Low-Low Cluster Area: With a total area of $163,890.440 \mathrm{~km}^{2}$, these areas are mainly distributed in the Chengdu Plain represented by Chengdu, Hilly Areas of Sichuan Basin represented by Neijiang, as well as a few places of Panzhihua in southern Sichuan, totally accounts for $33.72 \%$ of the province's land area. The Low-Low Cluster Area is characterized by plains and shallow hills with high regional economic level. these areas havefewer geological disasters accompanied by strong regional disaster capacity and low disasters-caused poverty risk.

(3) The areas of High-Low Outlier and Low-High Outlier are $152.63 \mathrm{~km}^{2}$ and $798.17 \mathrm{~km}^{2}$ respectively, accounting for $0.03 \%$ and $0.16 \%$ of the province's land area, respectively. 
Those two area type are mainly scattered in the borders between High-High and Low-Low Cluster Areas.

\section{Conclusions and Discussion}

Based on the quantitative evaluation of geological disaster risk and regional development, the poverty risk caused by disasters and their spatial differentiation characteristics were explored in Sichuan Province as the research interest. The results are as following.

(1) The overall risk of geological disasters in Sichuan Province is relatively high, with high and relatively high risk areas accounting for $43.34 \%$, low and relatively low risk areas accounting for $29.86 \%$, and medium risk areas accounting for $26.80 \%$. Due to the topography, geological structure, human activities and other factors, the risk of geological disasters has obvious spatial clustering and differentiation. The risk of geological disasters in Mountain and Ravine Area is significantly higher than that in other areas, while the risk of geological disasters in Chengdu Plain and its eastern adjacent areas is significantly low.

(2) In recent years, the medical care, education security, infrastructure construction and other improvements in Sichuan Province have been improved gradually. the residents' income and the efficiency of resource utilization have been improved constantly.the national economy and society are developing steadily. The percentage of areas in High and Relatively High development level is $31.70 \%$, the percentage of Medium level areas accounts for $33.33 \%$. the percentage of Low and Relatively Low areas is $34.97 \%$. The spatial differences of regional development level are prominent.the overall development of high altitude areas and remote mountain areas in western Sichuan is low on the whole, while the development level of plain areas and hilly areas are of high advantages.

(3) The area proportion of the poverty risk grades caused by disasters in Sichuan Province shows the characteristics of medium risk area $>$ relatively low risk area $>$ relatively high risk area $>$ low risk area $>$ high risk area.the spatial pattern shows the characteristic of "high in the west and low in the east". There was a significant positive spatial correlation between poverty risk and disasters. High-High Cluster mainly distributed in Garze Tibetan Autonomous Prefecture, Liangshan Yi Autonomous Prefecture and other areas in western Sichuan and southwestern Sichuan, accounts for 29.30\% of the total area. Low-Low Cluster areas are mainly distributed in Chengdu Plain and Hilly Areas of Sichuan Basin, accounting for 33.72\% of the total area; High-Low and Low-High Outlier Areas are small and scattered randomly.

(4) There is a geospatial coupling between regional development level and geological disaster risk in Sichuan Province. It is necessary to coordinate society and economic development with disaster reduction and prevention management, develop ecological agriculture and green industries according to local conditions. it is also important to improve the industrial structure towards the direction of green and sustainable development, thus reduce the dependence on natural resources and enhance the ability to resist disasters from the regional and personal perspectives, to ensure the coordinated development of economy, society and ecology.

\section{Ethical Approval}


The paper meets the ethical requirements and standards.

\section{Consent to Participate}

Not applicable.

\section{Consent to Publish}

The author agrees to participate in the publication of the paper

\section{Data availability statement}

The data that support the findings of this studyare available upon reasonable request from theAuthors.

\section{Acknowledgment}

The authors would like to thank the reviewers for their valuable suggestions on the manuscript. This research was funded by the National Natural Science Foundation of China (No. 42071232), Supported by Sichuan Science and Technology Program (2020YFS0308) and Open Foundation of the Research Center for Human Geography of Tibetan Plateau and Its Eastern Slope (Chengdu University of Technology).

\section{Conflicts of interest}

The authors declare that they have no competing interests.

\section{Authors' contributions}

Writing - review and editing, Mingshun Xiang; formal analysis and investigation, Jin Yang; investigation and validation, linsen Duan and Wenheng Li; resources, Fengran Wei; data curation, Wenbo Yang and Chunjian Wang; supervision, Qiuchi Deng.

\section{ORCID iD}

mingshun xiang https://orcid.org/0000-0002-3156-4808

\section{References}

Alkire S and Foster J (2011) Counting and multidimensional poverty measurement Journal of Public Economics 95:476-487.https://doi.org/10.1016/j.jpubeco.2010.11.006

Andrew B and Mikhail V C (2017) Climate change vulnerability in the food, energy, and water nexus: concerns for agricultural production in Arizona and its urban export supply Environmental Research Letters 12:1-13. https://doi.org/10.1088/17489326/aa5e6d

Barbier E B (2010) Poverty, development, and environment Environment and Development Economics 15:635-660. https://doi.org/10.1017/S1355770X1000032X

Berberich K (2019) Representations of Poverty and Place: Using Geographical Text Analysis to Understand Discourse International Journal of Corpus Linguistics 24:548-553. https://doi.org/10.1075/ijcl.00019.ber

Besagni G and Borgarello M (2019) The socio-demographic and geographical dimensions of fuel poverty in Italy Energy Research \& Social Science 49:192-203. 
https://doi.org/10.1016/j.erss.2018.11.007

Bidisha S H, Mahmood T and Hossain M B (2021) Assessing Food Poverty, Vulnerability and Food Consumption Inequality in the Context of COVID-19: A Case of Bangladesh Social Indicators Research 4:1-24. https://doi.org/10.1007/s11205-020-02596-1

Bird K and Shepherd A (2003) Livelihoods and chronic poverty in Semiarid Zimbabwe World Development 31:591-610. https://doi.org/10.1016/s0305-750x(02)00220-6

Carter M R and Barrett C B (2006) The economics of poverty traps and persistent poverty: an asset-based approach The Journal of Development Studies 42:178-199. https://doi.org/10.1080/00220380500405261

Chen M G, Lu D D and Zhang H (2009) Comprehensive Evaluation and the Driving Factors of China's Urbanization Acta Geographica Sinica 64:387398. https://doi.org/10.11821/xb200904001

Cui P (2014) Progress and prospects in research on mountain hazards in China Progress in Geography 33:145-152. https://doi.org/10.11820/dlkxjz.2014.02.001

Ding W G, Wei Y L, Wang L K, Mi X and Ye W F (2013) Integrated relationship among environmental degradation, disasters and poverty in Gansu Province Journal of Arid Land Resources and Environment 27:1-7 https://doi.org/10.13448/j.cnki.jalre.2013.03.001

Elwood S, Lawson V and Sheppard E (2017) Geographical relational poverty studies Progress in Human Geography 41:745-765. https://doi.org/10.1177/0309132516659706

Gignoux J and Menéndez M (2016) Benefit in the wake of disaster: Long-run effects of earthquakes on welfare in rural Indonesia Journal of Development Economics 118:26-44. https://doi.org/10.1016/j.jdeveco.2015.08.004

He X D, Mai X M and Shen G Q (2021) Poverty and Physical Geographic Factors: An Empirical Analysis of Sichuan Province Using the GWR Model SUSTAINABILITY 13:100(01)-100(17). https://doi.org/10.3390/su13010100

Iparraguirre J L (2012) Geographical distribution of disability living allowance and attendance allowance and income poverty Journal of Maps 8:189-193. https://doi.org/10.1080/17445647.2012.695441

Jessie A W, Kerrie A W, Nicola K A, Malcolm N, David L A, Rebecca K R, Nina T, Kerrie L $M$ and Erik M (2016) Rising floodwaters: mapping impacts and perceptions of flooding in Indonesian Borneo Environmental Research Letters 11:1-15. https://doi.org/10.1088/1748-9326/11/6/064016

Jin G, Deng X Z, Dong Y and Wu F (2020) China's multidimensional poverty measurement and its spatio-temporal interaction characteristics in the perspective of development geography Acta Geographica Sinica 75:1633-1646. https://doi.org/10.11821/dlxb202008006

Kirby R H, Reams M A, Lam N, Zou L, Dekker G G J and Fundter D Q P (2019) Assessing Social Vulnerability to Flood Hazards in the Dutch Province of Zeeland International Journal of Disaster Risk ence 10:233-243. https://doi.org/10.1007/s13753-019-0222-0

Li X H, Zhou Y and Chen Y F (2020) Theory and measurement of regional multidimensional poverty Acta Geographica Sinica 75:753-768. https://doi.org/10.11821/dlxb202004007

Li Y H, Wu W H and Liu Y S (2020) Evolution of Global Major Disasters During Past 
Century and Its Enlightenments to Human Resilience Building Bulletin of Chinese Academy of Sciences 35:345-352. https://doi.org/10.16418/j.issn.1000-3045.20200220003

Liang L P, Liu Y G, Tang Z H, Zou Q and Li J J (2019) Geologic hazards susceptibility assessment based on weighted information value Bulletin of Soil and Water Conservation 39:176-182. https://doi.org/10.13961/j.cnki.stbctb.2019.06.026

Liu Y L, Liao H P, Cai J, Li J, Li T, Zhu L and He T (2020) Spatio-temporal coupling relationship between multi-functionality of land use and multidimensional poverty in China's southwestern mountainous areas China population, resources and environment 30:154-164. https://doi.org/10.12062/cpre. 20200641

Luo L G, Pei X J, Gu H, He Y H and Liang J (2020) Risk assessment of geohazards induced by " 8.8 " earthquake based on GIS in Jiuzhaigou scenic area Journal of Natural Disasters 29:193-202. https://doi.org/10.13577/j.jnd.2020.0321

Ni J P, Li P, Wei C F and Xie D L (2009) Potentialities evaluation of regional land consolidation based on AHP and entropy weight method Transactions of the Chinese Society of Agricultural Engineering 25:202-209. https://doi.org/10.3969/j.issn.10026819.2009.05.38

Rampengan M M F, Boedhihartono A K, Law L, Gaillard J C and Sayer J (2014) Capacities in Facing Natural Hazards: A Small Island Perspective International Journal of Disaster Risk Science 5:247-264. https://doi.org/10.1007/s13753-014-0031-4

Sakai Y, Estudillo J P, Fuwa N, Higuchi Y, Sawada Y (2017) Do natural disasters affect the poor disproportionately? Price change and welfare impact in the aftermath of typhoon Milenyo in the rural Philippines World Development 94:16-26. https://doi.org/10.1016/j.worlddev.2016.12.036

Schleicher J, Schaafsma M, Burgess N D, Burgess C and Sandbrook F (2018) Poorer without it? The neglected role of the natural environment in poverty and wellbeing Sustainable Development 26:83-98. https://doi.org/10.1002/sd.1692

Shortliffe E H and Buchanan B G (1987) A model of inexact reasoning in medicine Mathematical Biosciences 23:351-379. https://doi.org/10.1016/0025-5564(75)90047-4

Tian H L and Zhang J Q (2016) Analyses of poverty risk induced by mountain disasters: a case study in Enshi, China Journal of Geo-information Science 18:307-314. https://doi.org/10.3724/SP.J.1047.2016.00307

Wu J D, Han G Y, Zhou H J and Li N (2018) Economic development and declining vulnerability to climate-related disasters in China Environmental Research Letters 13:110. https://doi.org/10.1088/1748-9326/aaabd7

Xu D D, Peng L, Liu S Q, Su C J, Wang X X and Chen T T (2017) Influences of Sense of Place on Farming Households' Relocation Willingness in Areas Threatened by Geological Disasters: Evidence from China International Journal of Disaster Risk Science 8:16-32. https://doi.org/10.1007/s13753-017-0112-2

Zhou Y, Li X H, Tong C Y and Huang H (2021) The geographical pattern and differentiational mechanism of rural poverty in China Acta Geographical Sinica 76:903920. https://doi.org/10.11821/dlxb202104009 